\title{
Déconstruire la politique européenne de gestion des déchets nucléaires
}

Une stratégie critique?

Grégoire Lits

\begin{abstract}
Dans le champ scientifique, les catégories employées apparaissent souvent, presque par définition, épistémologiquement objectives et moralement neutres. Or, des domaines comme l'environnement ou la santé publique comptent aujourd'hui à coup sûr parmi les lieux de confrontation politique les plus engagés. Dans cet essai, nous examinons les catégories structurant la dernière Proposition de Directive européenne sur le traitement des déchets nucléaires, afin de mettre au jour les acteurs engagés dans cette législation et les lieux de sa discussion. Une telle analyse mettra en avant le caractère immédiatement politique des catégories mobilisées dans cette problématique pourtant éminemment technique.

Mots-clefs: catégories, déchets nucléaires, Union Européenne, espace discursif, controverse socio-technique

More often than not, categories structuring techno-scientific problems appear to us as epistemologically objective and morally neutral. However, it also seems that issues such as the environment or the struggle for public health are amongst the most politically charged ones. In this essay we examine the categories that structure the recent Proposal for a Council Directive on the management of spent fuel and radioactive waste, in order to identify the important actors of this field and their spaces of discussion. Such an analysis should highlight the political dimension of the seemingly unbiased categories that are put forward in this technical debate.
\end{abstract}

Keywords: categories, radioactive waste, European Union, discourse, socio-technical controversy

\section{Catégories et constructivisme}

Toute politique se base sur un jeu de catégories construites, condition nécessaire à toute perception et toute action. C'est en insistant sur l'importance, tant éthique que méthodologique, de la prise en compte de ce constat que Stéphane Baele ouvre ce numéro d'Émulations. Cette interpellation constructiviste sur les catégories langagières n'est bien sûr pas nouvelle dans les sciences sociales. En sociologie, dans le milieu des années 1990, Philippe Corcuff a d'ailleurs fait du « constructivisme social» le critère de convergence des «nouvelles sociologies» rassemblées autour de ce nouvel espace 
commun de questionnement (CORCUFF 1995, p.6). À la même époque, Didier Fassin publiait dans la Revue française de sociologie un article qui, en s'intéressant à la question de l'apparition des nouvelles pauvretés urbaines en France, aux Etats-Unis et en Amérique latine, a parfaitement synthétisé le projet d'une sociologie constructiviste centrée sur les catégories langagières au travers d'une problématisation qui

«vise à montrer comment des notions s'imposent dans l'espace public, comment elles se transforment souvent imperceptiblement, comment elles agissent en retour sur ce qu'elles prétendent désigner en toute neutralité. Il s'agit en somme de faire apparaître les enjeux sociologiques des modes de représentation et de qualification du monde social » (FASSIN 1996, p.67).

À partir de ce projet centré sur l'étude des catégories, Fassin proposait un programme de recherche visant à mettre au jour la manière dont une société, via l'adoption des catégories qu'elle utilise pour décrire sa pauvreté, définit de «nouvelles topologies sociales ». Dans cette conception, un des projets originels de la sociologie (dresser la carte de la différenciation sociale existant au sein d'un espace social intégré) passait par l'étude de la genèse et des effets des catégories langagières utilisées dans l'espace social. À cet égard, l'enjeu de ce numéro est certes de renouveler cette manière constructiviste d'interroger le politique. Mais bien plus que cela, il est de voir d'une part, que nous ne pouvons plus éthiquement ignorer la dimension constructiviste dans nos analyses ${ }^{1}$, et, d'autre part, qu'il nous faut interroger ce qui, dans nos manières de problématiser, est tributaire de cette perspective.

Dans ce contexte, nous essayons ici de rendre compte de certains aspects constructivistes qui peuvent enrichir une analyse des politiques publiques de gestion des déchets radioactifs en Belgique $e^{2}$. Plus spécialement, le but de ce court article est de présenter une des différentes manières dont sont catégorisés en Belgique les déchets radioactifs, afin d'évaluer la pertinence de ce point de départ pour comprendre la façon dont s'articulent les différentes échelles de décision politique qui s'y rapportent. Dit simplement, nous posons la question de savoir s'il est possible de comprendre la topologie sociale (pour reprendre les termes de Fassin) d'une décision publique à partir de l'analyse des catégories langagières qui y sont mobilisées. Pour ce faire, nous serons amené à examiner un des nouveaux documents de référence dans le domaine du traitement des déchets nucléaires: la proposition de Directive européenne intitulée

${ }^{1}$ De la même manière qu'il n'était possible il y a trente ans de ne pas se positionner face au marxisme (CAille \& Chanial 2009). Par ailleurs l'auteur remercie Stéphane Baele pour le dialogue à partir duquel cet article s'est construit.

2 Plus précisément, il s'agit ici pour nous d'un travail de mise au point sur les soubassements constructivistes du travail d'enquête à réaliser dans le cadre de notre recherche de doctorat. 
«Proposal for a Council Directive on the management of spent fuel and radioactive waste $»^{3}$ (COMMISSION EUROPEENNE 2010).

\section{Une conception praxéologique et interactionnelle du discours et de l'espace public}

Pour répondre à cette question, il faut avant tout identifier les espaces ${ }^{4}$ dans lesquels est utilisé et défini le jeu de catégories qui nous intéresse - celui des déchets radioactifs, qui constituent déjà une première catégorie - et qui est relié d'une manière ou d'une autre au processus décisionnel qui nous intéresse - celui déterminant le mode de gestion des déchets radioactifs qui sera adopté en Belgique. Ces espaces ne sont pas des lieux géographiques (ils n'épousent d'ailleurs pas les frontières de la Belgique), mais sont plutôt à comprendre comme des configurations discursives et praxéologiques ${ }^{5}$ : des groupements (ou des réseaux) d'individus interagissant et communicant dans le contexte particulier du débat nucléaire. En cela, l'analyse des catégories socio-politiques peut s'inscrire, d'une certaine manière, dans la lignée des travaux sur la démocratie délibérative (e.g. HABERMAS 1997). Quant à la nécessité éthique des catégories, évoquée supra, elle pourrait alors être conçue comme un complément critique à l'éthique de la discussion.

L'identification des espaces en question passe donc par une première enquête visant à localiser les «endroits» où les acteurs sont susceptibles, en agissant, d'influencer la décision finale autant que le mode de décision lui-même. En d'autres termes, l'identification des espaces de la décision passe par l'identification des inter-actions de la participation à la décision, c'est-à-dire par une cartographie de la topologie sociale des acteurs $^{6}$ engagés dans la décision. Cet engagement est principalement accessible quand il se fait dans un espace discursif public (i.e. un espace discursif qui produit des traces

\footnotetext{
${ }^{3}$ Disponible sur le site web de la Commission:

http://ec.europa.eu/energy/nuclear/waste_management/doc/2010_11_03_proposal_directive_r adiactive_waste.pdf

${ }^{4}$ La notion d'espace est utilisée dans cet article de manière métaphorique. Elle ne désigne pas un lieu géographique, mais un lieu social interactionnel rassemblant des acteurs dans des pratiques discursives.

5 Pour une présentation approfondie d'une conception praxélogique et interactionelle des discours politique voir, à propos des politiques urbains : MONTDADA 2000.

${ }^{6}$ Le terme d'acteur est plurivoque. Il recouvre autant de simples individus que des organisations et institutions de natures et ordres divers. La différentiation de ces types d'acteurs en termes de capacité d'action est importante mais ne sera pas présentée dans cet article qui reste un exercice prospectif.
} 
matérielles $^{7}$ de la discussion qui sont publiées et peuvent être mobilisées par des personnes qui peuvent être extérieures à l'espace initial afin, à leur tour, de s'engager dans l'espace décisionnel) mais qui n'est pas forcément lié directement à l'espace public (en son sens commun) de l'Etat-nation qu'il concerne (ici, la Belgique) ${ }^{8}$. La double idée derrière cette conception est qu'un espace décisionnel ne se confond pas avec un seul espace discursif public (par exemple la Chambre des représentants, ou le Parlement européen), et que les espaces discursifs divers produisent des traces ${ }^{9}$ qui figent les discours et permettent la mobilisation de ces derniers pour l'action. Ces traces peuvent être par exemple des livres verts, des propositions de loi, des questions parlementaires, des articles d'ONG sur internet, des pages de groupes Facebook, des comptes-rendus de réunions, des publicités, des expositions didactiques, des actes de colloques scientifiques, des actions de désobéissance civile, des rapports d'expertise, des conventions internationales, des sondages d'opinion, des consultations citoyennes, etc. Ainsi, en ce qui concerne l'étude des catégories structurant le débat sur les déchets nucléaires en Belgique, initiée afin d'éclairer - si tant faire ce peut - la topologie sociale de cette thématique, l'analyse se doit de débuter par un examen minutieux des différents espaces discursifs où la problématique se construit en permanence.

Une fois ces traces, ces fixations de discours et de concepts repérées, il est alors possible 1) de remonter aux acteurs qui les ont produites et ainsi de reconstituer à rebours les différents espaces discursifs où elles sont mobilisées et dans lesquelles elles prennent sens, et 2) d'analyser les définitions particulières de la catégorie des « déchets radioactifs » (et autres catégories liées) qu'elles véhiculent. Dans cet article, nous nous concentrons sur l'analyse d'une nouvelle trace qui pourra avoir un impact fondamental sur le processus décisionnel belge: la Proposition de Directive européenne sur la gestion des déchets radioactifs et du combustible usé, rendue publique le 3 novembre 2010 par le Commissaire européen chargé de l'énergie Günther Oettinger.

\footnotetext{
7 Cette idée renvoie à la notion de «mobile immuable» telle que définie par Bruno Latour (2006b, pp.11-16).

${ }^{8}$ L'existence d'engagements non publicisés (par exemple certaines actions de lobbysme) doit également pouvoir être prise en compte. Dans ce cas les actions à la base de la publicisation de certains discours et de certaines positions sont très difficilement traçables, mais devront bien, pour avoir un effet sur la décision, ressortir à un moment donné dans des discours et prises de positions publiques.

9 Pour une présentation de la méthode de description d'une situation de controverse basée sur l'analyse des traces laissées par les acteurs, voir la présentation de l'Actor-Network theory systématisée par Bruno Latour (LATOUR 2006a).
} 


\section{Une première trace : la Proposition de Directive européenne sur la gestion des déchets radioactifs}

Le 3 novembre 2010, le commissaire Günther Oettinger a annoncé dans une conférence de presse le dépôt d'une proposition de directive intitulée « Proposal for a Council Directive on the management of spent fuel and radioactive waste» (COMMISSION EUROPEENNE 2010). Cette proposition de directive a pour objectif : « de créer un cadre juridiquement contraignant à l'échelle de l'UE pour garantir que tous les États membres appliquent les normes communes développées au sein de l'Agence internationale de l'énergie atomique (AIEA) pour toutes les étapes de la gestion du combustible usé et des déchets radioactifs jusqu'à leur stockage définitif. »10 (Commission Européenne 2010b)

Cette proposition n'est pas la première du genre. Elle fait suite à une première proposition portant de manière conjointe sur la gestion des déchets et sur des questions générales de sureté des installations nucléaires, adoptée par la Commission en 2003 (COMMISSION EUROPEENNE 2003) mais n'ayant pas été finalisée malgré certaines propositions d'amendements. À l'évidence, étant donné que toute proposition de directive européenne constitue un cadre juridique potentiellement contraignant pour l'État belge, les catégories qui structurent le document de 2010 ont une importance considérable pour les acteurs du nucléaire, ce qui a eu pour effet, comme à l'habitude, de déclencher une activité de lobbying autour des termes structurant le texte ${ }^{11}$.

\subsection{Première catégorisation : déchets radioactifs /combustible irradié}

Le premier point de cette nouvelle proposition de directive («1.1. Background and objectives of the proposal ») définit directement deux objets distincts qui devront faire l'objet des mêmes procédures de gestion décrites par la suite. Ce premier système de catégorie distingue les « radioactive waste » (déchet radioactif) du «spent fuel» (combustible irradié). Cette distinction annoncée dès le titre de la directive se retrouve développée dans les premiers paragraphes du mémorandum explicatif qui précède le corps du texte :

\footnotetext{
10 Communiqué de presse de la Commission : Reference: IP/10/1460 Date: 03/11/2010 : http://europa.eu/rapid/pressReleasesAction.do?reference $=I P / 10 / 1460 \&$ format $=$ HTML\&aged $=0 \&$ language $=\mathrm{FR} \&$ guiLanguage $=\mathrm{en}$

11 Comme le rappellent Boessen et Maarse (2009), les caractéristiques formelle (son droit d'initiative) et informelle (son rôle d'établissement de réseaux d'expertise) de la Commission européenne en font une cible privilégiée du lobbying.
} 
«(...) The proposed Directive sets out an EU legal framework for spent fuel and radioactive waste management. It revises the Commission proposal for a Council (Euratom) Directive on the management of spent fuel and radioactive waste.

All Member States have radioactive waste. It is generated by many beneficial activities, such as electricity production in nuclear power plants and a range of radioisotope applications in medicine, industry, agriculture, research and education. The operation of nuclear reactors also generates spent fuel. There are currently two options for managing the spent (i.e. irradiated) fuel: reprocessing to recover plutonium and uranium for possible re-use, or interim storage and eventual direct disposal if the spent fuel is considered as waste as part of national policy. However, even if spent fuel is reprocessed, there is still 'ultimate waste', i.e. the separated vitrified residues containing the unrecycled fraction, that also needs to be disposed of. » (COM(2010) 618 final : 2, notre emphase)

Elle peut être schématisée de la manière suivante (fig.1) :

Fig. 1. Distinction entre déchet radioactif ( « radioactive waste ») et combustible irradié (« spent fuel »)

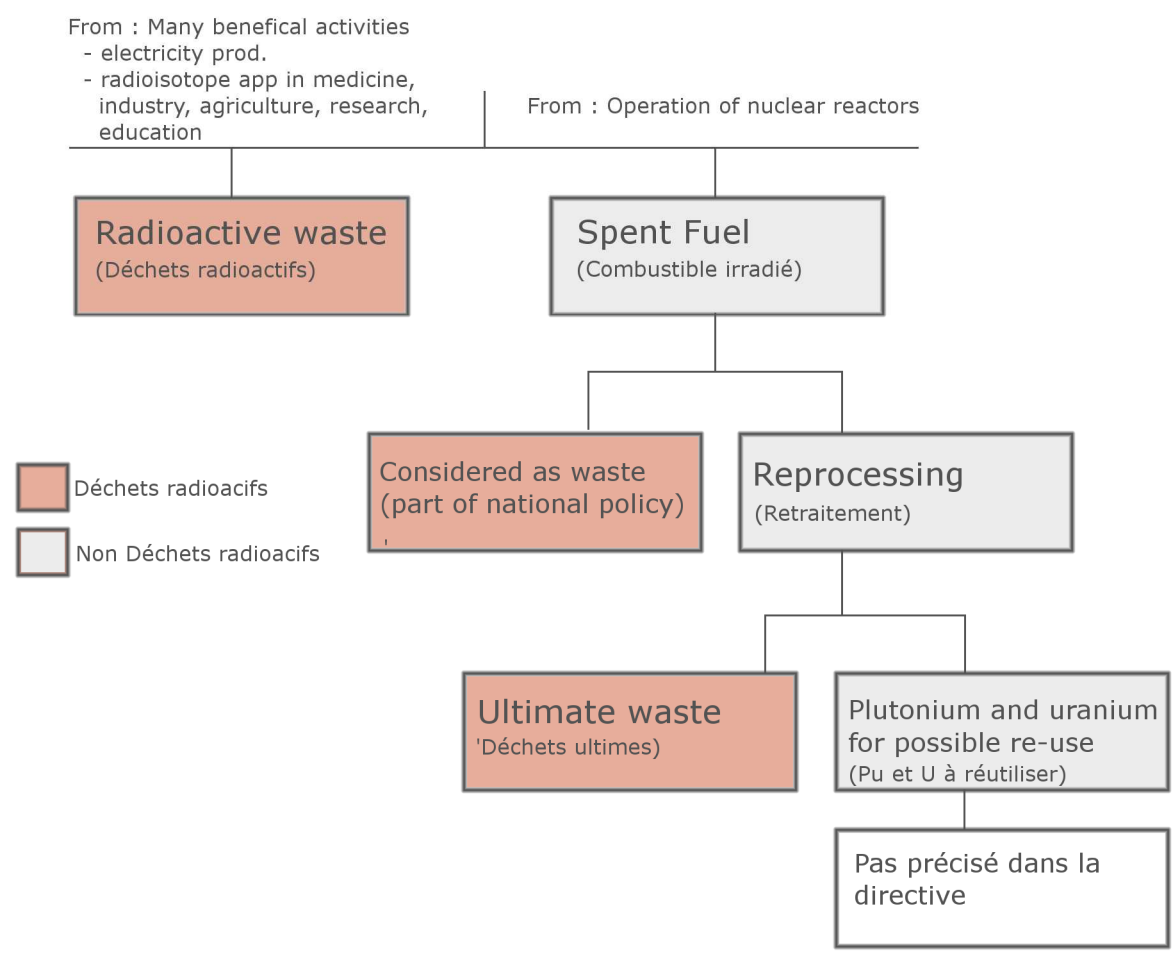


Cette distinction est reprise formellement dans l'article 3 de la proposition de directive ("Article 3. Definitions ») et servira donc de définition de référence en Europe si le texte est adopté. Les deux définitions de catégories suivantes sont donc primordiales :

«'radioactive waste' means radioactive material in gaseous, liquid or solid form for which no further use is foreseen by the Member State or by a natural or legal person whose decision is accepted by the Member State, and which is controlled as radioactive waste by a competent regulatory authority under the legislative and regulatory framework of the Member State; $(\operatorname{COM}(2010) 618$ final : 16)

«'spent fuel' means nuclear fuel that has been irradiated in and permanently removed from a reactor core; spent fuel may either be considered as a usable resource that can be reprocessed or be destined for disposal if regarded as radioactive waste; $(\operatorname{COM}(2010) 618$ final : 16)

\subsection{Seconde catégorisation : LILW/HLW}

A cette première catégorisation basée sur la provenance des déchets et sur les activités qui transforment des matières radioactives en déchet (e.g. activité de retraitement ou décision politique nationale) vient très rapidement s'y ajouter une deuxième qui est évoquée dans le même point 1.1. du mémorandum introductif et qui est déterminée quant à elle par des critères physiques et chimiques.

"Radioactive wastes are classified into low-, intermediate-, and high-level waste depending on their level of activity. A distinction can also be drawn between short-lived and long-lived radioactive wastes ${ }^{3}$. Short lived Low and Intermediate Level Waste (LILW) is typically disposed in near surface disposal facilities. For High Level Waste (HLW), by contrast, there is a world-wide scientific and technical consensus that deep geological disposal represents the safest and most sustainable option ${ }^{4}$.

Commission Recommendation of 15 September 1999 on a classification system for solid radioactive

3 Commission Recommendation of 15 September 1999 on a classification system for solid radioactive waste, OJ L 265, 13.10.1999, p.37.

4 OECD-NEA Radioactive Waste Management Committee: "Collective Statement on Moving Forward to Geological Disposal of Radioactive Waste ", ISBN 978-9264-99057-9» ((COM(2010) 618 final : 2-3, notre emphase) 
Ce deuxième mode de classification repose sur deux critères : la durée de vie des déchets (courte durée de vie ou longue durée de vie) ainsi que leur niveau de radioactivité (faible, intermédiaire ou haute) et lie ces deux types à deux modes distincts de gestions ${ }^{12}$.

Cette catégorisation renvoie explicitement à une recommandation de la Commission de 1999 (COMMISSION EUROPÉENNE 1999) concernant une classification commune en Europe des déchets radioactifs, cette dernière recommandation se basant elle-même sur la proposition faite par l'AIEA ${ }^{13}$ en $1994^{14}$. Un des éléments remarquables de cette dernière proposition est qu'elle se caractérise par une forte réflexivité de la part des rédacteurs en ce qui concerne l'action de catégoriser (8 pages sur 34 sont consacrées uniquement à cette question). Dans le document de l'AIEA, il est explicitement énoncé que proposer un mode de classement des déchets est une opération de construction reposant sur la sélection des critères à prendre en compte ainsi que sur la manière de structurer ces derniers :

«Classification is an approach which is used, mainly when the quantity of elements considered (objects or ideas) is large, to ease management of the elements by reducing their number. Classification is realized by selecting the main features (criteria) and by structuring these criteria.» (AIEA 1994, p.2, notre emphase)

Les rédacteurs y reconnaissent également que le classement qui va être opéré dépend du point de vue adopté ainsi que des objectifs recherchés, et va même jusqu'à reconnaitre la spécificité de leur proposition :

"Classification systems for radioactive waste may be derived from different points of view, such as safety related aspects, process engineering demands or regulatory issues. In this publication, emphasis is given to the safety related aspects, since these are in most cases of highest importance. This does not preclude the consideration of other aspects. » (AIEA 1994, p.3, notre emphase)

En reconnaissant ainsi la multiplicité des catégorisations possibles des déchets nucléaires, l'AIEA reconnaît du même coup, implicitement, que le champ pourtant hautement technique et scientifique du nucléaire ne peut échapper à une catégorisation politique, c'est-à-dire une catégorisation reflétant un choix sur l'organisation de la cité.

12 Cette liaison étant dans le premier cas justifiée par un argument traditionaliste : « [LILW] is typically disposed... », et, dans le second, par un argument d'autorité : "there is a world-wide scientific and technical consensus that... » (COMMISSION EuROPÉENNE 2010).

${ }_{13}$ Ce point y est affirmé clairement: "La classification internationale des colis de déchets radioactifs de l'Agence internationale de l'énergie atomique (AIEA) constitue une bonne base de départ pour l'établissement d'un système commun de classification à l'usage des États membres de l'Union européenne » (COMmission EuropeEnne 1999, p.4).

${ }^{14}$ IAEA (1994), document disponible sur

http://www-pub.iaea.org/MTCD/publications/PDF/Pub950e_web.pdf. 


\subsection{Premières remarques sur ces deux catégorisations}

La brève analyse de ces deux types de catégories nous permet déjà de souligner une caractéristique jouant un rôle dans la manière dont peut être mobilisé et véhiculé un set de catégories par des acteurs de la décision. Dans le premier cas (déchets radioactifs/combustible irradié), nous observons un jeu de catégories qui n'apparait pas comme le fruit d'un travail discursif de construction. Il n'est pas possible à la lecture unique de la proposition de directive de voir d'où vient cette catégorisation, ni de situer le point de vue - voire les objectifs - à partir duquel elle est construite. Le jeu de catégories apparaît comme une «boite noire» dans le discours : les opérations ayant mené à sa construction et qui in-forment la réalité dont on parle, ne sont pas explicitées. À l'inverse, le second type de catégorisation (LILW/HLW) présente très explicitement, via un jeu de références à différents textes antérieurs (c'est-à-dire à d'autres traces d'espaces discursifs), la généalogie des concepts employés. Dans ce second cas, le rédacteur de la proposition de directive donne des indications très claires au lecteur soucieux de pouvoir remonter aux sources des catégories mobilisées et par là même aux acteurs ayant forgé le cadre de compréhension utilisé.

\section{Critique de la directive}

Ce premier exercice nous permet donc de voir qu'avant même d'être mobilisée par d'autres acteurs du processus décisionnel, le texte et le jeu de catégories qu'il propose peut nous mener vers d'autres acteurs aux sources de ces catégories. Il nous faut maintenant considérer la suite, lorsque cette proposition est rendue publique et qu'elle peut dès lors être critiquée.

Plusieurs réactions ont émergé suite à la publication de la proposition. Ces réactions forment de nouvelles traces qu'il est possible de suivre pour agrandir la représentation de l'espace de la controverse. L'analyse des critiques proposées dans ces traces va nous permettre, d'une part, de voir la mesure dans laquelle la dimension construite des catégories utilisées dans la directive est questionnée par les acteurs, et, d'autre part, d'analyser la base sur laquelle ces critiques vont reposer. Dans le cadre de cet article, nous nous limitons à considérer deux critiques parmi les plus conséquentes de la proposition, émanant respectivement de Greenpeace et du Groupe parlementaire des Verts/ALE.

\subsection{La réaction d'une ONG internationale : Greenpeace}

Le 26 octobre 2010, Greenpeace diffuse un communiqué en réaction à la proposition imminente de la Commission (GREENPEACE 2010). Cette note lui adresse six critiques négatives et un point positif. Sur ces six critiques, cinq portent sur la manière dont la proposition se positionne vis-à-vis d'autres traces de la controverse ou par rapport à 
d'autres acteurs (les critiques 1, 2, 4, 5, 6). Le tableau ci-dessous en reprend les éléments centraux.

\begin{tabular}{|c|c|c|}
\hline Titre de la critique & Corps de l'argument & Acteurs ou traces liées \\
\hline 1. A PR exercice & $\begin{array}{l}\text { "The nuclear industry wants to over- } \\
\text { come public resistance to new nuclear } \\
\text { power stations. Greenpeace believes this } \\
\text { proposal was drafted with this aim in } \\
\text { mind » (p.1) }\end{array}$ & $\begin{array}{l}\text { - Le secteur de l'industrie nucléaire } \\
\text { - Le grand public }\end{array}$ \\
\hline 2. Dangerous & $\begin{array}{l}\text { "The Commission claims a scientific } \\
\text { consensus has been reached and con- } \\
\text { struction should proceed. However, it } \\
\text { makes no reference to scientific studies } \\
\text { and has not ordered a literature review of } \\
\text { research» (p.1) }\end{array}$ & - Les scientifiques \\
\hline 3. No Plan B & « No plan B proposed » & / \\
\hline $\begin{array}{l}\text { 4. NW in open } \\
\text { environments... }\end{array}$ & $\begin{array}{l}\text { "Fails to set standards for so-called 'au- } \\
\text { thorized emissions'» (p.1) «Uranium } \\
\text { mining wastes are not considered as ra- } \\
\text { dioactive waste. » }\end{array}$ & $\begin{array}{l}\text { - Autres directives de la } \\
\text { commission (Directive sur les } \\
\text { déchets de l'industrie extractive) }\end{array}$ \\
\hline $\begin{array}{l}\text { 5. Out of line with EU } \\
\text { Hazardous waste laws }\end{array}$ & $\begin{array}{l}\text { No « attempt to harmonize RW legisla- } \\
\text { tion with laws covering other hazardous } \\
\text { wastes » (p.2) }\end{array}$ & $\begin{array}{l}\text { - Autres directives de la } \\
\text { Commission (Directive sur les } \\
\text { déchets dangereux) }\end{array}$ \\
\hline $\begin{array}{l}\text { 6. Civil society } \\
\text { marginalised }\end{array}$ & $\begin{array}{l}\text { "Input from outside the nuclear industry } \\
\text { amounts to two out of } 17 \text { pages. Argu- } \\
\text { ments from environmentalists, con- } \\
\text { cerned citizens and local municipalities } \\
\text { were marginalised as stemming from a } \\
\text { "fundamental opposition towards nu- } \\
\text { clear energy" instead of being judged on } \\
\text { their merits » (p.2) }\end{array}$ & $\begin{array}{l}\text { - Les environnementalistes } \\
\text { - Les citoyens concernés } \\
\text { - Les municipalités locales }\end{array}$ \\
\hline
\end{tabular}

L'exposition de ces six critiques montre très clairement que Greenpeace, pour critiquer la directive, dévoile son caractère construit et les relations qu'elle noue avec d'autres acteurs et d'autres traces qui ont (ou auraient dû) être pris en compte dans l'argumentaire de la directive. Il faut noter que la critique $\mathrm{n}^{\circ} 4$ porte spécifiquement sur la définition des déchets radioactifs proposée par la directive - définition qui n'est, selon l'ONG, pas assez englobante puisqu'elle rejette hors du concept les déchets miniers. 


\subsection{La critique d'un parti politique européen : Le Groupe des Verts/ALE au Parlement européen}

La position des Verts a été défendue par la parlementaire Rebecca Harms, et fut notamment exposée au public lors de la séance de présentation d'une étude sur le sujet commandée par le Groupe des Verts (Nuclear Waste Management in the European Union) ${ }^{15}$. Le document en question est un texte de six pages diffusé à l'occasion de cette réunion ${ }^{16}$. Il comporte quatre paragraphes distincts correspondant à trois critiques différentes. Le tableau ci-dessous reprend ces dernières de façon synthétique.

\begin{tabular}{|c|c|c|}
\hline $\begin{array}{c}\text { Titre du } \\
\text { paragraphe }\end{array}$ & Corps de l'argument & $\begin{array}{l}\text { Acteurs ou } \\
\text { traces liées }\end{array}$ \\
\hline $\begin{array}{l}\text { Qu'est-ce qu'un } \\
\text { déchet radioactif } \\
\text { ? }\end{array}$ & $\begin{array}{l}\text { - La directive ne prend pas en compte l'ensemble des déchets } \\
\text { radioactifs (notamment les déchets miniers, militaires...). (p.2) } \\
\text { - «La définition des matières pour lesquels 'aucune utilisation } \\
\text { ultérieure n'est prévue' (voir définition des déchets radioactifs } \\
\text { proposés dans la directive supra) est clairement insuffisante. » } \\
\text { (p.2) } \\
\text { - « S'il y a une législation européenne sur les déchets } \\
\text { radioactifs, on ne peut s'en remettre aux États membres pour } \\
\text { décider si certaines catégories comme étant des déchets } \\
\text { radioactifs ou non [en faisant référence directement plus haut } \\
\text { dans le texte au fuel usagé] » (p.3) }\end{array}$ & $\begin{array}{l}\text { - ENSREG (voir } \\
\text { détail infra) }\end{array}$ \\
\hline $\begin{array}{l}\text { Qui décide quoi } \\
\text { et comment? }\end{array}$ & $\begin{array}{l}\text { «La Commission affirme qu'elle 'a procédé à de nombreuses } \\
\text { consultations via différentes initiatives à l'échelon européen'. Cependant, } \\
\text { la procédure de 'consultation publique ouverte' de la Commission } \\
\text { via le questionnaire en ligne est tout à fait insuffisante. En } \\
\text { outre, le fait que la plupart des commentaires et suggestions } \\
\text { formulés par des représentants de la société civiles n'ont pas } \\
\text { du tout été abordé dans la nouvelle proposition de directive } \\
\text { constitue une grave préoccupation. } \\
\text { Au contraire, il doit y avoir une évaluation exhaustive des idées } \\
\text { et opinions qui sont directement introduites dans le processus } \\
\text { décisionnel. L'institution d'un 'comité d'experts' composé } \\
\text { d'experts désignés par chaque États membres [...] est } \\
\text { insuffisante. » (p. 4) }\end{array}$ & $\begin{array}{l}\text { - Les experts } \\
\text { (ENSREG) } \\
\text { - Les États } \\
\text { membres } \\
\text { - La société civile }\end{array}$ \\
\hline
\end{tabular}

15 Cette présentation à eu lieu au Parlement européen de Bruxelles le 1er décembre 2010.

16 Cette étude est disponible en version anglaise sur le site www.kernenergiedebatte.de (consulté le $6 / 12 / 2010)$ :

http://www.kernenergiedebatte.de/pdfs/359735.hintergrundpapier_atommuellrichtlinie@en.pdf Pour un résumé de la critique, voir aussi sur le communiqué de presse du 3/11/2010 du Groupe des Verts: http://www.greens-efa.eu/cms/pressreleases/dok/359/359614nuclear_waste (a)en.htm. 


\begin{tabular}{|l|l|l|}
\hline $\begin{array}{l}\text { Qui paye la } \\
\text { note ? }\end{array}$ & $\begin{array}{l}\text { "dans sa résolution de 2007, le PE demande explicitement de } \\
\text { 'prendre en compte le principe du pollueur-payeur. } \\
\text { L'application de ce principe signifie que : } \\
\text { - l'évaluation des coûts doit être réalisée indépendamment des } \\
\text { opérateurs et de ceux qui assument les coûts » } \\
\text { - des fonds suffisants doivent être disponibles au moment où } 2007 \\
\text { ils sont nécessaires » (p. 4) }\end{array}$ & $\begin{array}{l}\text { du PE } \\
\text { - Les opérateurs qui } \\
\text { assument les coûts }\end{array}$ \\
\hline $\begin{array}{l}\text { Qui s'occupe des } \\
\text { questions non } \\
\text { résolues ? }\end{array}$ & $\begin{array}{l}\text { 4 questions de fond dans le débat ne sont pas abordées : } \\
\text { - le court terme/le long terme } \\
\text { - Environnement et sécurité (safety)/sécurité (security } \\
\text { concern) } \\
\text { - Récupérabilité et réversibilité } \\
\text { - Internationalisation poussée latente dans la directive }\end{array}$ & \\
\hline
\end{tabular}

Les critiques qui sont le plus longuement développées sont 1) celle qui porte explicitement sur la définition de « déchet radioactif» proposée dans la directive, et 2) celle qui adresse la possibilité laissée aux États membres de classer ou non le combustible usé comme déchet. Cette critique qui ouvre donc explicitement la «boite noire » que constitue la distinction déchet/combustible usé, est en outre appuyée par une mise en évidence du caractère construit de cette dichotomie. Le Groupe des Verts dévoile en effet, pour appuyer son propos, la définition que l'ENSREG (European Nuclear Safety Regulator Group) ${ }^{17}$ a proposé à la Commission et qui, selon les verts, est « encore plus inacceptable» (p.2) que celle posée dans la proposition de directive. Cette définition, qui se retrouve dans un document intitulé «ENSREG's suggestion for the content of a Directive on Sustainable Management of Radioactive Waste and Spent Fuel », est globalement la même que celle de la directive - à un mot près qui attire l'attention du Groupe des Verts :

«Matières radioactives sous forme gazeuse, liquide ou solide pour lesquelles aucune utilisation ultérieure n'est prévue ou envisagée et qui font l'objet d'un contrôle en tant que déchets radioactifs dans le cadre législatif et réglementaire de l'État membre concerné» (The Greens - European Free Alliance \& Harms 2010, p.2, emphase originale dans la publication).

17 Ce groupe associe l'ensemble des «autorités nationales de régulation» compétentes pour la gestion des déchets radioactifs dans chaque État membre. Il a été crée en 2007 par le Conseil de l'Europe sur recommandation de la Commission suite à l'échec de la première proposition de directive en 2004. Ses objectifs sont résumés de la manière suivante sur leur site web : «The role of ENSREG : All EU Member States that operate nuclear installations follow the basic principles set internationally for assuring nuclear safety and the safe management of radioactive waste and spent fuel. These principles are established in a series of international conventions. ENSREG's role is to help establish the conditions for continuous improvement and to reach a common understanding in these areas » (http://www.ensreg.eu/members-glance, consulté le 6 décembre 2010). 
Nous savons que la Commission a pris en compte l'avis soumis par l'ENSREG lors des consultations des parties prenantes. Le texte le mentionne très clairement dans le point 2 ( "Consultation of interested parties and impact assesment») du mémorandum explicatif introduisant la proposition de directive. Il y est en effet stipulé que :

«The consultation involved governments, national regulators, radioactive waste management organizations, radioactive waste producers and others in the Member States, together with the various European Institutions, non-governmental organizations and other partners. A detailed contribution from the European Nuclear Safety Regulators Group (ENSREG) was taken into account. » (COMMission EuROPEENNE 2010, p.6, notre emphase)

La Commission reconnait donc avoir pris en compte les recommandations d'ENSREG mais ne dit pas clairement sur quelles parties de la proposition elles ont porté. Les Verts, en faisant référence à cette suggestion de définition faite par l'ENSREG et en montrant le décalage entre cette proposition et la définition finalement présente dans le projet de directive, ouvrent la «boite noire» de la catégorisation déchet/combustible usé, ce qui leur permet de mettre en question son caractère inquestionable. La critique des Verts ne se limite donc pas a dire que la catégorisation déchet/combustible n'est pas adéquate, elle va plus loin en attaquant la manière dont cette catégorisation et la directive en général a été construite en posant la question : «qui décide quoi et comment?».

\section{Conclusions}

Bien que partielle (nous n'analysons que deux critiques de la proposition et deux sets de catégorie), faisant état d'une recherche en train de se faire et donc encore très peu formalisée, l'analyse de la nouvelle Proposition de Directive sur les déchets radioactifs nous permet d'illustrer le fait qu'il existe différentes manières, pour un législateur, de mobiliser et de véhiculer des sets de catégories construits en amont du projet de législation.

L'analyse rapide de deux des principales critiques du document nous permet par ailleurs de nous rendre compte que ce caractère construit des catégories non seulement n'échappe pas aux groupements qui exercent cette critique, mais qu'en plus de cela l'utilisation par le législateur de catégories «boîtes noires ", va permettre aux critiques de se développer très facilement et d'attaquer en quelques mouvements l'entièreté de la proposition en dévoilant les opérations de construction qu'elle laisse implicite. Ce constat nous amène donc à penser que la possible énonciation d'une éthique constructiviste de la délibération politique n'est pas qu'une invention théorique de chercheurs coupés du monde. La déconstruction des «boîtes noires » est déjà une stratégie de critique exercée dans les espaces publics discursifs contemporains.

Étudier les politiques de gestion des déchets nucléaires au travers des catégories qui sont mobilisées pour structurer ce débat, c'est donc comprendre que même dans un champ hautement technique, scientifique, et donc largement perçu comme «objectif » 
et «neutre» par le grand public, les concepts sont des enjeux politiques. Dit simplement, il s'agit de comprendre que toute catégorie est politique, ou que, d'une certaine façon, les catégories sont le lieu fondamental des relations de pouvoir. En somme, une telle analyse met à mal l'idéal de décisions purement technocratiques, dénuées de toute dimension normative, et par là même renforce les études des catégories présentes dans les débats où leur artefactualité et leur charge morale apparaissent de manière plus immédiate (politique en matière de migration, politiques sociales, etc.). En complément d'une analyse des «usages politiques de l'expertise» (BOSWELL 2010), la mise à jour d'une topologie sociale particulière via les catégories qui y sont mobilisées contribue par conséquent à rendre plus transparente la complexité des relations de pouvoir qui prennent nécessairement place autour d'un enjeu scientifique géré dans la sphère publique. Et, par là même, ouvre peut-être la voie à une éthique de la discussion plus performante.

\section{Bibliographie}

Boessen S., MAARse H. (2009): «A ban on tobacco advertising: The role of interest groups ». In COEN D., RICHARDSON J., ed. (2009): Lobbying the European Union: Institutions, actors, and issues. Oxford: Oxford University Press.

BOSWELL C. (2010): The political use of expert knowledge. Cambridge: Cambridge University Press.

Caillé A., Chanial Ph. (2009): "Présentation ». In Revue du MAUSS - Que penser de Marx aujourd'bui ?, vol.2 n³4, pp5-25.

COMmission EuRopeEnNe (1999): Recommandation de la Commission du 15 septembre 1999 relative à un système de classification des déchets radioactifs solides. Bruxelles, 15 septembre 1999, SEC(1999) 1302 final.

Commission EuropeEnNe (2003): Proposition de Directive (Euratom) du Conseil sur la gestion du combustible nucléaire irradié et des déchets radioactifs. Bruxelles, 30 janvier 2003, COM 2003/32 final. Version amendée : COM (2004)526 final.

Commission EuropéEnne (2010): Proposal for a Council Directive on the management of spent fuel and radioactive waste. Bruxelles, 3 novembre 2010, $\operatorname{COM}(2010) 618$ final.

COMMission EuropéENNE (2010b): Communiqué de presse. Bruxelles, 3 novembre 2010, $\mathrm{IP} / 10 / 1460$.

CORCUFF Ph. (1995): Les nomvelles sociologies. Paris: Nathan Université.

FASSIN D. (1996): «Exclusion, underclass, marginalidad. Figures contemporaines de la pauvreté urbaine en France, aux Etats-Unis et en Amérique Latine ». In Revue française de sociologie, vol. $37 \mathrm{n}^{\circ} 1, \mathrm{pp} 37-75$. 
Greenpeace (2010): Proposed Nuclear waste directive - A Greenpeace overview. Document online, disponible sur www.greenpeace.org.

HABERMAS J. (1997): Droit et démocratie. Entre faits et normes. Paris: Gallimard.

IAEA (1994): Classification of Radioactive Waste. A Safety Guide. Document online, disponible sur http://www-pub.iaea.org/MTCD/publications/PDF/ Pub950e_ web.pdf .

LATOUR B. (2006A): Changer de société, refaire de la sociologie. Paris: La Découverte/Poche.

LATOUR B. (2006B): «Les 'vues' de l'esprit. Une introduction à l'anthropologie des sciences et des techniques ». In Latour B., CAllon M., AKrich M., Sociologie de la traduction. Textes fondateurs. Paris: Presses de l'école des mines de Paris, pp. 33-70.

MONDADA L. (2000), « Pratiques discursives et configuration de l'espace », In LEVY J., Lussault M., ed., Logiques de l'espace, esprit des lieux, Paris, Belin, pp. 165-175.

The Greens - European Free Alliance, Harms R. (2010): Ce que les Verts/ALE attendent de la directive européenne sur la gestion du combustible usé et des déchets radioactifs. Working document diffusé lors d'une réunion d'information au Parlement Européen le $1^{\text {er }}$ décembre 2010 